\title{
Learning the Roots of Visual Domain Shift
}

\author{
Tatiana Tommasi ${ }^{1(\otimes)}$, Martina Lanzi ${ }^{2}$, Paolo Russo $^{2}$, and Barbara Caputo ${ }^{2}$ \\ ${ }^{1}$ University of North Carolina at Chapel Hill, Chapel Hill, NC, USA \\ ttommasi@cs.unc.edu \\ 2 Department of Computer, Control and Management Engineering, \\ University of Rome La Sapienza, Rome, Italy \\ \{lanzi, russo, caputo\}@dis. uniroma1.it
}

\begin{abstract}
In this paper we focus on the spatial nature of visual domain shift, attempting to learn where domain adaptation originates in each given image of the source and target set. We borrow concepts and techniques from the CNN visualization literature, and learn domainness maps able to localize the degree of domain specificity in images. We derive from these maps features related to different domainness levels, and we show that by considering them as a preprocessing step for a domain adaptation algorithm, the final classification performance is strongly improved. Combined with the whole image representation, these features provide state of the art results on the Office dataset.
\end{abstract}

Keywords: Domain adaptation $\cdot$ CNN visualization

\section{Introduction}

In 2010 Saenko et al. imported the notion of domain adaptation from natural language processing to visual recognition [17]. They showed how training visual classifiers on data acquired in a given setting, and testing them in different scenarios, leads to poor performance because the training and test data belong to different visual domains. Since then, domain adaptation has become a widely researched topic. The vastly dominant trend is to summarize images into global features (being them handcrafted BoWs or the most modern CNN-activation values) and remove the domain shift through an optimization problem over feature data points distributions. This strategy is theoretically sound and effective, as it has been largely demonstrated over the years. To give a quantitative estimate of the progress in the field, one might look at the accuracy values obtained over the Office-31 dataset, a data collection presented in [17] and quickly become the domain adaptation reference benchmark: performance has increased on average from $27.8 \%$ [8] to $72.9 \%$ in only three years [16]. While such progress is certainly impressive, it is not fully clear that it is coupled with an equally deepened knowledge of the roots of domain shift.

We believe the time is ripe for gaining a better understanding of how visual concepts such as illumination conditions, image resolution or background give 


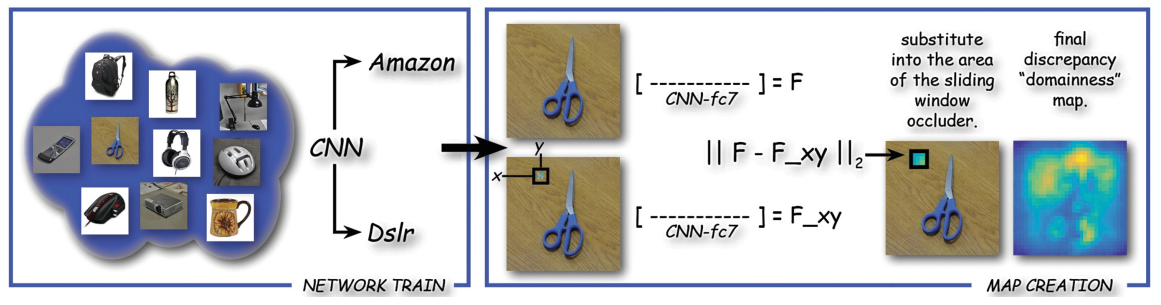

Fig. 1. A CNN network is initially trained for domain classification. The obtained model is used as feature extractor both for the original and the occluded images. The difference among the obtained representation is saved and visualized in the image area, creating the domainnes map. Yellow/Blue indicate areas at high/low domainness. Best viewed in color. (Color figure online)

rise to the domain shift. As these visual concepts often have a spatial connotation - more or less illuminated parts of images, informative object parts that are more or less visible, etc. - our goal is to localize the domain shift in the source and target data, or at least to spatially ground it. Is it doable? and if yes, what do we learn from it? Could it be used to improve the effectiveness of any domain adaptation algorithm?

This paper attempts to answer these questions. We first show that by learning to classify visual domains (binary classification on source-target domain pairs), it is possible to obtain domain localization maps as a byproduct, where high/low map values indicate high/low domain specificity (Fig. 1, Sect.3). We dub the score used to define the map domainness. By analyzing the domainnes map we are able to evaluate the correlation between domain-specificity and object-specificity. Depending on the domain-pairs we can identify when the domain shift come mostly from the background and when instead it involves the objects (Sect.4). Armed with this knowledge, we create 3 different features from each image: a low-domainness feature, a mid-domainness feature and a high-domainness feature (Fig. 3, Sect.5). With this strategy each domain-pair becomes a set of 9 pairs. We show that by applying domain adaptation over each pair and then recombining the results through high level integration, we systematically achieve a substantial increase in performance as opposed to previously reported results obtained by the same methods on the whole images. This approach enables us to obtain the new state of the art on the Office-31 dataset for unsupervised domain adaptation.

\section{Related Work}

Domain Adaptation. The goal of domain adaptation is to compensate the variation among two data distributions, allowing to reuse information acquired from a source domain on a new, different but related, target domain. Some techniques perform this by simply re-weighting or selecting the samples in the source domain $[6,15]$, or clustering them to search for visually coherent sub-domains 
$[7,10]$. Other approaches modify existing source classifiers to make them suitable for the target task $[1,2]$, or search for transformations that map the source distribution into the target one [3,9]. Different strategies propose to learn both a classification model and a feature transformation jointly. Few of them rely on SVM-like risk minimization objectives and shallow representation models [4,11], while more recent approaches leverage over deep learning [16, 19,20].

Despite their specific differences, all these methods consider the whole image as a data unit, corresponding to a sample drawn from a given domain distribution. Some work has been recently done for dealing directly with image patches within the NBNN framework [14,18] with promising results. Here we push research further in the direction of relating domain adaptation and spatial localities in images: we study how the domain information is distributed inside each image, and how to deal with domain-specific and domain-generic image patches.

CNN Visual Analysis. A number of works have focused on understanding the representation learned by CNNs. A visualization technique which reveals the input stimuli that excite individual feature maps was introduced in [22]. Girshick et al. [5] visualized which patches within a dataset are the most responsible for strong activations at higher layers in the model. Simonyan et al. [13] demonstrated how saliency maps can be obtained from CNN by projecting back from the fully connected layers of the network. Zhou et al. [23] focused on scene images, and by visualizing the representation learned by each unit of the network, they showed that object detectors are implicitly learned.

Inspired by these works we introduce an image mask-out procedure to visualize what a domain classification network learns and how the domain information is spatially distributed. We are not aware of previous work attempting to learn what part of images are more or less responsible for the domain shift.

\section{Domainness Prediction}

Given the images of a source/target domain pair we resize them to $256 \times 256$, and we randomly split them into a training and test set. On the training set we learn a CNN for domain recognition: specifically we initialize the parameters of conv1-fc7 using the released CaffeNet [12] weights and we then further finetuned the network for binary classification on the domain labels. The test set is extended by replicating each image many times with small random occluders at different locations: we use $16 \times 16$ image patches positioned on a dense grid with stride 8 . This results in about 1000 occluded images per original image. Finally we feed both the original and the occluded test images into the defined network and we record the difference between their respective fc7 activation values (4096dimensional output of the seventh fully connected layer after ReLu). The $L_{2}-$ norm of this difference is spatially saved in the image inside the occluder area and the obtained value for overlapping occluders is averaged defining a smooth discrepancy map with values rescaled in $\{0,1\}$ (see Fig. 1 ). We call it domainness map: an area of high domainness corresponds to a region that highly influences 


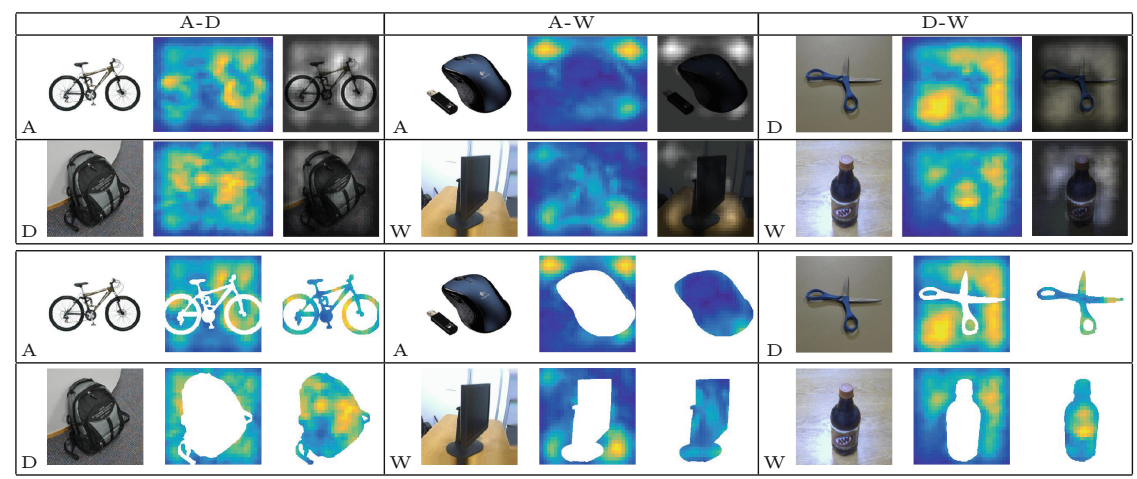

Fig. 2. Top: each table element shows the original image (left), the domainness map (center) and the map-image overlap (right - low domainness regions occluded). Column titles indicate the domain-pairs. Bottom: original image (left), the background part of domainness map (center) and the foreground part of the map (right) when using the segmentation masks. Best viewed in color. (Color figure online)

the final domain choice for the image, and thus it can be considered as domainspecific. On the other hand, an area of low domainness appears to be less relevant for domain recognition, hence more domain-generic. Note that the procedure we propose is unsupervised with respect to the object classes depicted in the source and target images.

\section{Domainness Analysis}

To have a better understanding of the information captured by the domainness maps we analyze here how the domainness distribution in each image relates with image foreground and background areas.

We use the standard Office-31 dataset [17] which collects images from three distinct domains, Amazon (A), Dslr (D) and Webcam (W). The first contains images downloaded from online merchants and mainly present white background, while the second and the third are acquired respectively with a high resolution DSL camera and with a low resolution webcam in real settings with background and lighting variations. The 31 categories in the dataset consist of objects commonly encountered in office settings, such as keyboards, scissors, monitors and telephones. We define train/test split of respectively 3000/612, 3000/315 and 1000/293 images for the A-W, A-D and W-D pairs and we follow the procedure described in the Sect. 3 to generate a map for each test image. Some of the obtained maps are shown in Fig. 2 - top.

By using object image masks obtained by manual segmentation we evaluated the average domainness value inside and outside the objects. Specifically, we focus on the central $227 \times 227$ area of the image to avoid artifacts that might be due to the CNN architecture used. Our evaluation reveals that for A-D and 


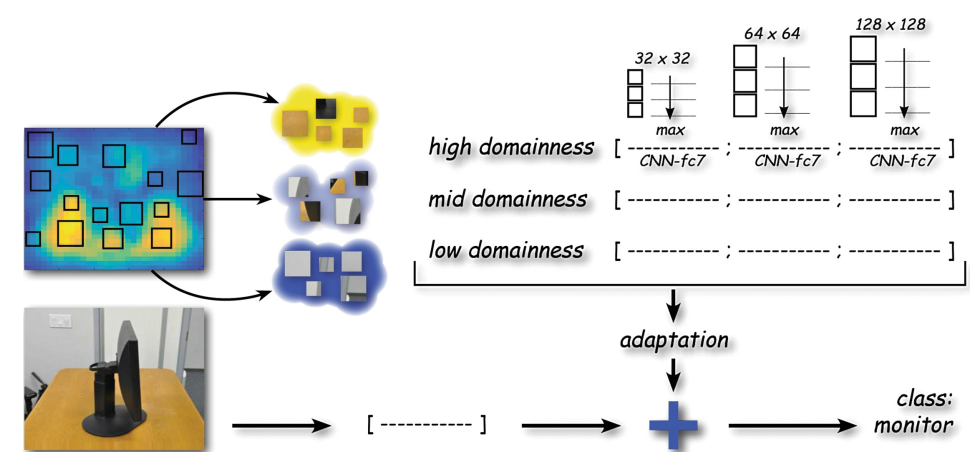

Fig. 3. Patches of three different dimensions are cropped from an image and organized according to their domainness score. CNN-fc7 features are extracted from the patches, max pooled and stacked. Classifiers are trained and tested using the obtained representation and the whole image. Confidence scores are combined for the final prediction.

D-W pairs the average domainness value is actually higher inside the objects (respectively 0.48 and 0.44$)$ than outside $(0.43,0.41)$. This indicates that most of the domain specific information tend to appear within the foreground rather than in the background part of the image. On the other way round, for A-W the background is the main responsible of domain shift, with an average domainness value of 0.24 against 0.27 obtained for the object area (see Fig. 2 - bottom).

\section{Exploiting Domainness Levels for Adaptation}

Finally we use the domainness maps to guide domain adaptation (see Fig. 3). Information from the images at three domainness levels (DL) are collected through local feature extraction. We start by sampling 100 patches of sizes $32 \times 32$ randomly from each image and associating to each one its average domainness. We then treat the patches as samples of the domainness distribution and identify its 33 th and 66 th percentiles. This allows to divide the patches into three groups with low- (L), mid- (M) and high-domainness (H). We follow [21] and used the CNN Caffe implementation pre-trained on Imagenet to collect fc7 features for each patch. Maximum pooling (element-wise maximum) is applied separately over the patches collected at different domainness levels. The procedure is repeated separately over other two image scales using patches of dimension $64 \times 64$ and $128 \times 128$. As a result, each image is represented by three feature vectors, each with 12288 elements, obtained by stacking the max pooled features at scale 32, 64 and 128. Besides these per-domainness-level descriptors we extracted fc7 features on the whole image as global representation both with (G-FT) and without $(\mathrm{G})$ fine tuning on the source.

How good are the descriptors? We perform object classification with linear SVM on all the domain pairs of the Office-31 dataset when using each DL descriptor 
Table 1. Accuracy on Office-31, unsupervised setting - full protocol.

\begin{tabular}{|c|c|c|c|c|c|c|c|}
\hline & $\mathrm{A} \rightarrow \mathrm{W}$ & $\mathrm{A} \rightarrow \mathrm{D}$ & $\mathrm{W} \rightarrow \mathrm{A}$ & $\mathrm{W} \rightarrow \mathrm{D}$ & $\mathrm{D} \rightarrow \mathrm{A}$ & $\mathrm{D} \rightarrow \mathrm{W}$ & Average \\
\hline G & 58.1 & 62.0 & 48.6 & 99.4 & 47.9 & 95.8 & 68.6 \\
\hline G-FT & 60.2 & 60.0 & 49.4 & 99.2 & 49.2 & 95.9 & 69.0 \\
\hline \multicolumn{8}{|c|}{ Domainness levels (DL) } \\
\hline L-L level & 44.1 & 49.0 & 28.1 & 90.2 & 32.0 & 84.8 & 54.7 \\
\hline L-H level & 41.6 & 42.2 & 27.9 & 77.8 & 30.9 & 73.6 & 49.0 \\
\hline L-M level & 48.9 & 51.0 & 29.9 & 90.8 & 34.5 & 87.4 & 57.1 \\
\hline M-M level & 53.0 & 52.1 & 33.3 & 94.1 & 35.1 & 88.6 & 59.4 \\
\hline M-L level & 45.3 & 50.0 & 31.8 & 89.4 & 31.4 & 84.0 & 55.3 \\
\hline M-H level & 47.1 & 47.9 & 29.3 & 88.8 & 31.6 & 83.1 & 54.6 \\
\hline H-H level & 46.2 & 44.1 & 28.9 & 90.4 & 31.6 & 81.6 & 53.8 \\
\hline H-L level & 44.1 & 42.6 & 31.0 & 83.3 & 30.3 & 76.5 & 51.3 \\
\hline H-M level & 52.4 & 47.4 & 33.1 & 92.5 & 35.1 & 85.7 & 57.7 \\
\hline \multicolumn{8}{|c|}{ Applying domain adaptation machine on domainness levels (DAM-DL) } \\
\hline L-L level & 40.8 & 50.0 & 28.4 & 99.9 & 32.9 & 88.9 & 55.7 \\
\hline L-H level & 42.8 & 44.5 & 28.6 & 82.5 & 32.2 & 81.0 & 51.9 \\
\hline L-M level & 48.3 & 50.6 & 31.5 & 92.8 & 34.5 & 91.7 & 58.3 \\
\hline M-M level & 51.5 & 51.9 & 35.1 & 96.9 & 38.2 & 92.2 & 60.9 \\
\hline M-L level & 41.8 & 48.8 & 33.7 & 93.8 & 33.8 & 87.5 & 56.6 \\
\hline M-H level & 47.8 & 48.3 & 31.4 & 93.2 & 35.5 & 87.3 & 57.3 \\
\hline $\mathrm{H}-\mathrm{H}$ level & 42.7 & 45.8 & 31.2 & 93.2 & 34.3 & 84.3 & 55.2 \\
\hline H-L level & 40.2 & 42.7 & 32.9 & 85.5 & 31.8 & 77.8 & 51.8 \\
\hline H-M level & 47.8 & 50.6 & 34.9 & 94.3 & 36.4 & 87.9 & 58.7 \\
\hline \multicolumn{8}{|c|}{ Combining domainness levels and whole image classification } \\
\hline $\mathrm{G}+\mathrm{DL}$ & $70.6 \pm 0.9$ & $74.9 \pm 1.1$ & $53.5 \pm 0.3$ & $100.0 \pm 0.1$ & $54.5 \pm 0.5$ & $98.3 \pm 0.1$ & 75.3 \\
\hline $\mathrm{G}+\mathrm{DAM}-\mathrm{DL}$ & $70.6 \pm 1.3$ & $\mathbf{7 6 . 9} \pm 0.4$ & $54.5 \pm 0.2$ & $100.0 \pm 0.1$ & $\mathbf{5 6 . 6} \pm 0.5$ & $\mathbf{9 9 . 5} \pm 0.1$ & 76.3 \\
\hline $\mathrm{G}-\mathrm{FT}+\mathrm{DL}$ & $\mathbf{7 1 . 5} \pm 0.6$ & $74.8 \pm 1.2$ & $54.0 \pm 0.1$ & $100.0 \pm 0.1$ & $55.8 \pm 0.8$ & $97.9 \pm 0.3$ & 75.7 \\
\hline G-FT + DAM-DL & $71.3 \pm 1.1$ & $75.3 \pm 1.0$ & $\mathbf{5 5 . 4} \pm 0.3$ & $100.0 \pm 0.1$ & $55.2 \pm 0.7$ & $98.9 \pm 0.3$ & 76.3 \\
\hline $\mathrm{DDC}[20]$ & $61.8 \pm 0.4$ & $64.4 \pm 0.3$ & $52.2 \pm 0.4$ & $98.5 \pm 0.4$ & $52.1 \pm 0.8$ & $95.0 \pm 0.5$ & 70.6 \\
\hline DAN [16] & $68.5 \pm 0.4$ & $67.0 \pm 0.4$ & $53.1 \pm 0.3$ & $99.0 \pm 0.2$ & $54.0 \pm 0.4$ & $96.0 \pm 0.3$ & 72.9 \\
\hline
\end{tabular}

to represent the images. We consider all the source labeled data as training samples and all the unlabeled target images define our test set (full protocol). The results are reported in the top part of Table1, together with the performance obtained using the whole image representation. The obtained classification accuracies indicate $\mathrm{M}$ as the most informative level. Although by construction $\mathrm{L}$ is the level which capture the most domain-generic cues, we speculate that $\mathrm{M}$ works best at balancing domain-generic and object-specific information.

Can we adapt across DLs? We use the Domain Adaptation Machine (DAM, [1]) to reduce the discrepancy across domainness levels. The results in the central part of Table 1 show an average accuracy improvement which ranges in $0.5-3 \%$ with respect to the previous results, confirming that adaptive techniques are beneficial.

Are DLs complementary to the whole image? We believe that different domainness levels provide complementary knowledge useful to solve domain adaptation. To test this hypothesis we integrate the per-class confidence score provided by the classifiers trained over DLs with that obtained when training on the whole image. 
Let's indicate with $j=1 \ldots 9$ the different DL pairs and with $c=1 \ldots C$ the object classes. Once we have all the margins $D_{c}^{j}$ obtained by separate-level classification and the margin $D_{c}^{G}$ obtained from the whole image we perform the final prediction with $c^{*}=\operatorname{argmax}_{c}\left\{\frac{1}{9} \sum_{j=1}^{9} D_{c}^{j}+D_{c}^{G}\right\}$. The obtained results (Table 1 - bottom part) compare favorably against the current state of the art CNN-architectures $[16,20]$ created on purpose to overcome visual domain shift.

\section{Conclusion}

The goal of this paper is to identify the spatial roots of visual domain shift. To this end we learned domainness maps from source and target data which are able to localize the image parts more or less responsible for the domain shift. We proved experimentally that generating features from image regions with different degrees of domainness and feeding them to a domain adaptation algorithm leads to a significant boost in performance. Moreover, in combination with whole image features, they allow to obtain state of the art results on the Office dataset.

\section{References}

1. Duan, L., Tsang, I.W., Xu, D., Chua, T.S.: Domain adaptation from multiple sources via auxiliary classifiers. In: International Conference on Machine Learning - ICML (2009)

2. Duan, L., Tsang, I.W., Xu, D., Maybank, S.J.: Domain transfer svm for video concept detection. In: IEEE International Conference on Computer Vision and Pattern Recognition - CVPR (2009)

3. Fernando, B., Habrard, A., Sebban, M., Tuytelaars, T.: Unsupervised visual domain adaptation using subspace alignment. In: International Conference in Computer Vision - ICCV (2013)

4. Fernando, B., Tommasi, T., Tuytelaars, T.: Joint cross-domain classification and subspace learning for unsupervised adaptation. Pattern Recogn. Lett. 65, 60-66 (2015)

5. Girshick, R., Donahue, J., Darrell, T., Malik, J.: Rich feature hierarchies for accurate object detection and semantic segmentation. In: IEEE Conference on Computer Vision and Pattern Recognition - CVPR (2014)

6. Gong, B., Grauman, K., Sha, F.: Connecting the dots with landmarks: discriminatively learning domain-invariant features for unsupervised domain adaptation. In: International Conference on Machine Learning - ICML (2013)

7. Gong, B., Grauman, K., Sha, F.: Reshaping visual datasets for domain adaptation. In: Advances in Neural Information Processing Systems - NIPS (2013)

8. Gong, B., Shi, Y., Sha, F., Grauman, K.: Geodesic flow kernel for unsupervised domain adaptation. In: IEEE Conference on Computer Vision and Pattern Recognition - CVPR (2012)

9. Gretton, A., Borgwardt, K.M., Rasch, M.J., Schölkopf, B., Smola, A.: A kernel two-sample test. J. Mach. Learn. Res. 13(1), 723-773 (2012) 
10. Hoffman, J., Kulis, B., Darrell, T., Saenko, K.: Discovering latent domains for multisource domain adaptation. In: Fitzgibbon, A., Lazebnik, S., Perona, P., Sato, Y., Schmid, C. (eds.) ECCV 2012. LNCS, vol. 7578, pp. 702-715. Springer, Heidelberg (2012). doi:10.1007/978-3-642-33709-3_50

11. Hoffman, J., Rodner, E., Donahue, J., Saenko, K., Darrell, T.: Efficient learning of domain-invariant image representations. In: International Conference on Learning Representations - ICLR (2013)

12. Jia, Y., Shelhamer, E., Donahue, J., Karayev, S., Long, J., Girshick, R., Guadarrama, S., Darrell, T.: Caffe: convolutional architecture for fast feature embedding. arXiv preprint abs/1408.5093 (2014)

13. Simonyan, K., Vedaldi, A., Zisserman, A.: Deep inside convolutional networks: visualising image classification models and saliency maps. In: ICLR Workshop (2014)

14. Kuzborskij, I., Carlucci, F.M., Caputo, B.: When naïve bayes nearest neighbours meet convolutional neural networks. In: IEEE Conference on Computer Vision and Pattern Recognition - CVPR (2016)

15. Lim, J.J., Salakhutdinov, R., Torralba, A.: Transfer learning by borrowing examples for multiclass object detection. In: Neural Information Processing Systems NIPS (2011)

16. Long, M., Cao, Y., Wang, J., Jordan, M.: Learning transferable features with deep adaptation networks. In: International Conference on Machine Learning - ICML (2015)

17. Saenko, K., Kulis, B., Fritz, M., Darrell, T.: Adapting visual category models to new domains. In: Daniilidis, K., Maragos, P., Paragios, N. (eds.) ECCV 2010. LNCS, vol. 6316, pp. 213-226. Springer, Heidelberg (2010). doi:10.1007/ 978-3-642-15561-1_16

18. Tommasi, T., Caputo, B.: Frustratingly easy NBNN domain adaptation. In: International Conference on Computer Vision - ICCV (2013)

19. Tzeng, E., Hoffman, J., Darrell, T., Saenko, K.: Simultaneous deep transfer across domains and tasks. In: International Conference in Computer Vision - ICCV (2015)

20. Tzeng, E., Hoffman, J., Zhang, N., Saenko, K., Darrell, T.: Deep domain confusion: maximizing for domain invariance. arXiv preprint abs/1412.3474 (2014)

21. Gong, Y., Wang, L., Guo, R., Lazebnik, S.: Multi-scale orderless pooling of deep convolutional activation features. In: Fleet, D., Pajdla, T., Schiele, B., Tuytelaars, T. (eds.) ECCV 2014. LNCS, vol. 8693, pp. 392-407. Springer, Heidelberg (2014). doi:10.1007/978-3-319-10584-0_26

22. Zeiler, M.D., Fergus, R.: Visualizing and understanding convolutional networks. In: Fleet, D., Pajdla, T., Schiele, B., Tuytelaars, T. (eds.) ECCV 2014. LNCS, vol. 8693, pp. 818-833. Springer, Heidelberg (2014). doi:10.1007/978-3-319-10590-1_53

23. Zhou, B., Khosla, A., Lapedriza, A., Oliva, A., Torralba, A.: Object detectors emerge in deep scene CNNs. In: International Conference on Learning Representations - ICLR (2015) 